\title{
ON A NEAR OPTIMAL SAMPLING STRATEGY FOR LEAST SQUARES POLYNOMIAL REGRESSION
}

\author{
YEONJONG SHIN AND DONGBIN XIU*
}

\begin{abstract}
We present a sampling strategy of least squares polynomial regression. The strategy combines two recently developed methods for least squares method: Christoffel least squares algorithm and quasi-optimal sampling. More specifically, our new strategy first choose samples from the pluripotential equilibrium measure and then re-order the samples by the quasi-optimal algorithm. A weighted least squares problem is solved on a (much) smaller sample set to obtain the regression result. It is then demonstrated that the new strategy results in a polynomial least squares method with high accuracy and robust stability at almost minimal number of samples.
\end{abstract}

Key words. Polynomial regression, least squares, christoffel function

1. Introduction. This paper is concerned with polynomial regression using least squares approach. As one of the most used methods in practice for approximating unknown functions, the performance of polynomial least squares regression depends on sample points, where data of the unknown function are collected. The choices of the samples typically follow two distinct approaches: random samples and deterministic samples. In random sampling, i.e., Monte Carlo (MC) method, one draws the samples from a probability measure, which is often defined by the underlying problem, whereas in deterministic sampling the samples follow certain fixed and deterministic rules to fill up the space systematically. For example, quasi Monte Carlo methods (QMC), lattice rules, orthogonal arrays, etc. Studies have been devoted to determine an "optimal" set, which aims to produce the best possible least squares solution with minimal number of points. Different design criteria have been proposed, resulting in a collection of optimality designs, e.g., A-optimality, D-optimality, V-optimality, etc. The study of these generally falls into the topic of design of experiments (DOE), see, for example, $[1,2,7,11,12,13,19,21]$, and the references therein. In a recent work of [20], a design criterion is derived by directly minimizing the difference between the finite set least squares solution and the ideal solution from a dense set. The results in the so-called quasi-optimal set.

The polynomial least squares solution is obtained by solving an over-determined linear system of equations for the coefficients. Let the size of the system be $M \times p$, where $M$ is the number of samples and $p$ is the number of unknown coefficients in the polynomial. Ideally, one would prefer $M$ to be as large as possible. However, since collecting sample data requires resources, small number of samples are preferred from a practical point of view. A well accepted rule-of-thumb is to use linear oversampling by letting $M=\alpha p$, where $\alpha=1.5 \sim 3$. On the other hand, some recent mathematical analysis revealed that such a linear over-sampling is asymptotically unstable for polynomial least squares using Monte Carlo sampling and quasi Monte Carlo, cf. $[8,16,15,14]$. In fact, for asymptotically stable polynomial regression, one should have at least $M \propto p \log p$ and in many cases $M \propto p^{2}$. We remark that these results are for bounded domains, i.e., $d$-dimensional hypercube. In a more recent work [17], a method termed Christoffel least squares (CLS) was proposed. Here one computes a weighted least squares problem, where the weights are derived from the

\footnotetext{
*Department of Mathematics, The Ohio State University, Columbus, OH 43210, USA. shin.481@osu.edu, xiu.16@osu.edu. This work is in part supported by AFOSR FA95501410022, DARPA N660011524053, and NSF DMS 1418771.
} 
Christoffel function of orthogonal polynomials. Instead of using the standard MC samples from the measure of the underlying problem, one samples instead from the pluripotential equilibrium measure. Analysis and extensive numerical tests in both bounded domain and unbounded domains were presented in [17] and demonstrated much improved stability property.

In this paper, we propose a sampling strategy for polynomial least squares regression by combining the two aforementioned recent developments from [20] and [17]. We first generate the samples by using the Christoffel least squares (CLS) method ([17]). This means that we re-formulate the problem into the weighted least squares polynomial regression and generate samples from the equilibrium measure (regardless of the measure defined by the problem). Prior to collecting the data at the samples, we then apply the subset selection criterion from [20] to determine the quasi-optimal subset, for any given number of samples specified by users. The data of the target function are then collected at this subset only and the corresponding weighted polynomial least squares problem is solved for the approximation of the target function. By doing so, the new strategy can produce, for any finite number of samples, the polynomial least squares regression result as close as possible to the CLS result obtained by an arbitrarily large number of samples, which has the near optimal stability property. In practice, this allows one to conduct the polynomial least squares regression using any given/affordable number of samples and obtain near the optimal approximation result. Moreover, the quasi-optimal subset selection from [20] can be implemented in a greedy manner, resulting in a ranking of the dense background samples from the CLS. This further allows one to add samples in a nested manner and obtain progressively better (and near optimal) approximation results. The combination of the quasi-optimal subset selection and the CLS method results in a near optimal sampling strategyu for least squares regression using polynomials. Our numerical results demonstrate the advantage of this strategy. The results also suggest that the major contribution for the performance improvement stems from the quasi-optimal subset selection.

Upon presenting the basic setup and a brief review of the CLS method ([17]) and the quasi-optimal subset selection ([20]) in Section 2, we present the procedure for the new near optimal sampling strategy in Section 3, along with some of its theoretical properties. Extensive numerical results are then presented in Section 4 to demonstrate the performance of the method.

2. Preliminaries. Consider a function $f(x)$, where $x \in D \subseteq \mathbb{R}^{d}, d \geq 1$. Let $y=$ $\left(y_{1}, \ldots, y_{M}\right)$, where $y_{i}=f\left(x_{i}\right), i=1, \ldots, M$, be the data vector. We are interested in approximating $f(x)$ using the data $y$. Here we consider polynomial approximation and let $\Pi$ be a polynomial space with $\operatorname{dim} \Pi=p$. Let $\phi_{i}(x), i=1, \cdots, p$, be a set of basis functions for $\Pi$ and

$$
q(x ; c)=\sum_{i=1}^{p} c_{i} \phi_{i}(x) .
$$

Requiring $q$ to approximate $f$ results in the following linear system of equations,

$$
A c=y,
$$

where $c=\left(c_{1}, \ldots, c_{p}\right)^{T}$ is the coefficient vector,

$$
A=\left(a_{i j}\right), \quad a_{i j}=\phi_{j}\left(x_{i}\right), \quad 1 \leq i \leq M, \quad 1 \leq j \leq p,
$$


is the model matrix. Here we focus on the over-determined regression problem with $M>p$. The standard least squares method seeks to minimize $\|A c-y\|^{2}$. Its solution is $c=\left(A^{T} A\right)^{-1} A^{T} y$. Throughout this paper all norms are the vector 2-norm, unless otherwise specified.

One can introduce weights in the least squares formulation. Let $W=\operatorname{diag}\left(w_{1}, \ldots, w_{M}\right)$ be a diagonal matrix with positive entries, a weighted least squares problem can be written as

$$
\min \|\sqrt{W} A c-\sqrt{W} y\|^{2}=\min \sum_{i=1}^{M} w_{i}\left(y_{i}-q_{n}\left(x_{i} ; c\right)\right)^{2} .
$$

Its solution is

$$
\hat{c}=\left(A^{T} W A\right)^{-1} A^{T} W y .
$$

This is the formulation we shall consider in this paper.

The polynomial space $\Pi$, where the approximant is sought, can be any properly defined polynomial spaces. Typically, a "degree" is associated with the space. Throughout this paper, we shall use $n \geq 1$ to denote such a "degree". The cardinality of the polynomial space, $p$, thus depends on $n$. For example, for the well known total degree polynomial space

$$
\mathcal{P}_{n}^{d}=\operatorname{span}\left\{x^{\alpha}=x_{1}^{\alpha_{1}} \cdots x_{d}^{\alpha_{d}}:|\alpha| \leq n\right\},
$$

where $\alpha=\left(\alpha_{1}, \ldots, \alpha_{d}\right)$ is multi-index with $|\alpha|=\alpha_{1}+\cdots+\alpha_{d}$, we have

$$
p=\operatorname{dim} \mathcal{P}_{n}^{d}=\left(\begin{array}{c}
n+d \\
d
\end{array}\right)=\frac{(n+d) !}{n ! d !} .
$$

For the full tensor polynomial space, $p=(n+1)^{d}$. Hereafter we will use subscript $n$ to denote the relation to the "degree" of the polynomial space whenever necessary and use the total degree polynomial space, unless otherwise noted.

Let us equip the domain $D \subseteq \mathbb{R}^{d}$ with a measure $\omega$, and denote $L_{\omega}^{2}(D)$ the weighted square integrable space. Given any $p$-dimensional polynomial subspace $\Pi_{n}$ of $L_{\omega}^{2}(D)$, let $\left\{\phi_{i}(x), i=1, \ldots, p\right\}$ be an orthonormal basis of $\Pi_{n}$, i.e.,

$$
\int_{D} \phi_{i}(x) \phi_{j}(x) \omega(x) d x=\delta_{i j}, \quad 0 \leq i, j \leq p .
$$

2.1. Christoffel least squares. We now briefly review the Christoffel least squares (CLS) method by [17]. It is a sampling strategy using the weighted least squares formulation (2.2) to achieve better theoretical properties than the standard least squares method using Monte Carlo sampling.

The "diagnoal" of the reproducing kernel of $\Pi_{n}$ is defined by

$$
K_{n}(x):=\sum_{i=1}^{p} \phi_{i}^{2}(x) .
$$

Note that $K_{n}$ does not depend on the choice of the orthonormal basis. The (normalized) Christoffel function from the theory of orthogonal polynomials ([18]) is

$$
\lambda_{n}(x):=\frac{p}{K_{n}(x)} .
$$


If $D$ is compact with nonvanishing interior and positive $d$-dimensional Lebesgue measure, then

$$
\lim _{n \rightarrow \infty} \lambda_{n}(x)=\frac{\omega(x)}{v(x)}, \quad \text { a.e. } \quad x \in D,
$$

where $v(x)$ is the Lebesgue weight function (a probability density) of the pluripotential equilibrium measure of $D([5])$

$$
d \mu_{D}(x)=v(x) d x
$$

Let

$$
\psi_{i}=\sqrt{\lambda_{n}(x)} \phi_{i}(x), \quad i=1, \ldots, p .
$$

They form a non-polynomial orthonormal basis satisfying

$$
\int_{D} \psi_{i}(x) \psi_{j}(x) \widetilde{v}_{n}(x) d z=\delta_{i j}, \quad \widetilde{v}(x)=\omega(x) / \lambda_{n}(x)
$$

In CLS, one uses the non-polynomial basis (2.11) and draw i.i.d. samples $\left\{x_{i}\right\}_{i=1}^{M}$ from the equilibrium measure (2.10), instead of the original measure $\omega(x)$. One then solves the weighted least squares problem (2.2) with

$$
W=\operatorname{diag}\left(w_{1}, \ldots, w_{M}\right), \quad w_{i}=\lambda_{n}\left(x_{i}\right), \quad\left\{x_{i}\right\}_{i=1}^{M} \sim \mu_{D} .
$$

For the bounded domain $D=[-1,1]^{d}$, its equilibrium measures is known to be the tensor product of the one-dimensional Chebyshev density, i.e.

$$
v(z)=\frac{d \mu_{D}}{d z}=\frac{1}{\pi \prod_{j=1}^{d} \sqrt{1-z_{j}^{2}}}, \quad z \in[-1,1]^{d} .
$$

For unbounded domains, their pluripotential equilibrium measures are not yet fully known. For a few special cases, the forms of the measures were conjectured in [17] and used in the CLS algorithm. More specifically, the following two cases were discussed.

- Tensor "half space" $D=[0, \infty)^{d} \subset \mathbb{R}^{d}$ with

$$
\omega(z)=\exp \left(-\sum_{j=1}^{d} z_{j}\right)
$$

The equilibrium measure is

$$
v(z)=\frac{d \mu_{D}}{d z}=C \sqrt{\frac{\left(4-\sum_{j=1}^{d} z_{j}\right)^{d}}{\prod_{j=1}^{d} z_{j}}}
$$

where $C$ is a normalization constant. The measure $\mu_{D}$ is supported only on the set $4 T^{d}$ with

$$
T^{d}=\left\{z \in \mathbb{R}^{d}\left|z_{j} \geq 0,\|z\|_{1}=\sum_{j=1}^{d}\right| z_{j} \mid \leq 1\right\} .
$$


- Whole space $D=\mathbb{R}^{d}$ with

$$
w(z)=\exp \left(-|z|^{2}\right) .
$$

The equilibrium measure is

$$
v(z)=\frac{d \mu_{D}}{d z}=C\left(2-|z|^{2}\right)^{d / 2},
$$

where $C$ is a normalization constant. The measure $\mu_{D}$ is supported only on the set $\sqrt{2} B^{d}$, where $B^{d}$ is the $d$-dimensional unit ball.

2.2. Quasi-optimal sample selection. We now review the quasi-optimal sample selection strategy developed in [20]. The strategy aims at selecting a subset of samples from a larger set of samples so that the least squares solution based on the subset has near optimal accuracy. Note that the selection is conducted prior to collecting the sample data $y$. Therefore, it is an nonadaptive approach that can identify the "important" subset samples prior to data collection. Note that the strategy is not restricted to polynomial regression.

Let $A_{M} \in \mathbb{R}^{M \times p}$ and $y_{M} \in \mathbb{R}^{M}$ be the model matrix and data vector, respectively, obtained using a large number of samples, $\Theta_{M}=\left\{x_{1}, \ldots, x_{M}\right\}$ with $M \gg 1$. The dense "candidate" set $\Theta_{M}$ can be arbitrarily chosen, random or deterministic. If the data vector $y_{M}$ is collected, then the least squares solution is readily available

$$
\hat{c}_{M}=\underset{c \in \mathbb{R}^{p}}{\operatorname{argmin}}\left\|A_{M} c-y_{M}\right\| .
$$

If, however, one is able to collect data only at a (much) smaller number of points $m<M$, then it is desirable to determine which $m$-point subset $\Theta_{m} \subset \Theta_{M}$ delivers better solution.

Let $S_{m}=\left[S_{(1)} ; \cdots ; S_{(m)}\right] \in \mathbb{R}^{m \times M}$ be a row selection matrix, with all rows having zero entries except a single entry of one at distinct locations. Then, selecting a subset samples $\Theta_{m}$ out of $\Theta_{M}$ is equivalent to selecting a subset rows $S_{m} A_{M}$. The quasi-optimal selection method from [20] seeks to maximize

$$
\mathcal{S}\left(S_{m} A_{M}\right),
$$

where for any $k \times p$ matrix $A$

$$
\mathcal{S}(A):=\left(\frac{\sqrt{\operatorname{det} A^{T} A}}{\prod_{i=1}^{p}\left\|A^{(i)}\right\|}\right)^{\frac{1}{p}} .
$$

Here $A^{(i)}$ denotes the $i$ th column of the matrix.

Maximization of the quantity $\mathcal{S}$ is motivated by the fact that the subset least squares solution

$$
\hat{c}_{m}\left(S_{m}\right)=\underset{c \in \mathbb{R}^{p}}{\operatorname{argmin}}\left\|S_{m} A_{M} c-S_{m} y_{M}\right\|
$$

should be as close as possible to the full least squares solution (2.18). The rigorous optimality can not be established without the knowledge of $y_{M}$. Therefore, the optimality (2.19) is termed quasi-optimal. Hereafter we will refer this sample selection criterion as S-optimality, for the ease of exposition. 
3. The near optimal sampling strategy. We now present the near optimal sampling strategy. It is a straightforward combination of the Christoffel least squares method (CLS) and the quasi-optimal point selection method (S-optimality), both of which are illustrated in the previous section.

3.1. Method. The near optimal sampling strategy consists of the following steps:

- For a given domain $D$ with a measure $\omega$, draw $M \gg 1$ number of i.i.d. random samples from the pluripotential equilibrium measure $\mu_{D}(2.10)$. If $D$ is unbounded, spread out samples by a scaling factor $n^{1 / t}$. Here $n$ is the degree of the polynomial and $t \geq 1$ is homogeneity exponent. For the whole space $\mathbb{R}^{d}$ with Gaussian measure, $t=2$; for the tensor half space $[0, \infty)^{d}$ with exponential measure, $t=1$. Detail of this scaling can be found in [17].

- Construct the weighted model matrix $\sqrt{W} A_{M}$.

- For any given number $m<M$, identify the quasi-optimal subset by applying the S-optimality strategy $(2.19)$ on the weighted model matrix $\sqrt{W} A_{M}$.

- Solve the weighted least squares problem (2.2) but only to the subset problem with $m$ points.

In another word, we solve the following problem

$$
\hat{c}_{m}=\underset{c \in \mathbb{R}^{p}}{\operatorname{argmin}}\left\|S_{m}^{\dagger} \sqrt{W} A_{M} c-S_{m}^{\dagger} \sqrt{W} y_{M}\right\|,
$$

where $A_{M}$ is the model matrix constructed by sampling the non-polynomial orthonormal basis (2.11) at $M$ i.i.d. samples from the equilibrium measure $\mu_{D}(2.10), W$ is the diagonal weight matrix (2.13), and the quasi-optimal row selection $S_{m}^{\dagger}$ is obtained by solving the S-optimality problem

$$
S_{m}^{\dagger}=\underset{S_{m}}{\operatorname{argmax}} \mathcal{S}\left(S_{m} \sqrt{W} A_{M}\right),
$$

where $\mathcal{S}$ is the quantity defined in (2.20). We again remark that the entire procedure for solving (3.2) is independent of the data vector $y_{M}$. Upon conducting the i.i.d. samples from $\mu_{D}$, one solves (3.2) to decide the $m$-point subset. It is only after this step that one is required to collect the function data at the $m$ select points to obtain $S_{m}^{\dagger} y_{M}$. Therefore, the problem (3.1) is a "smaller" least squares problem of size $m \times p$, and the full data vector $y_{M}$ is never required.

It is worth noting that the construction of the weighted model matrix $\sqrt{W} A_{M}$ can be easily accomplished by normalizing each row of the original unweighted model matrix $A_{M}$ and then rescaling it by a factor of $\sqrt{p}$, i.e.,

$$
A_{(i)} \rightarrow \sqrt{p} \frac{A_{(i)}}{\left\|A_{(i)}\right\|}, \quad i=1, \ldots, M .
$$

This is because each row of the weighted model matrix $\sqrt{W} A_{M}$ is

$$
\sqrt{w_{i}} A_{(i)}=\frac{\sqrt{p}}{\sqrt{K_{n}\left(x_{i}\right)}} A_{(i)}, \quad i=1, \ldots, M,
$$

where

$$
\left\|A_{(i)}\right\|=\sqrt{\sum_{k=1}^{p} \phi_{k}\left(x_{i}\right)^{2}}=\sqrt{K_{n}\left(x_{i}\right)} .
$$


We remark that by using the CLS method, the full size $M$-point model matrix for the least squares problem achieves much better stability, as detailed by [17]. Once the much better conditioned model matrix is constructed by the CLS method, the S-optimality strategy ([20]) allows one to determine the near optimal smaller subset with $m$ points such that the $m$-point least squares solution is almost the closest to the (unavailable) full set CLS solution. It is in this sense that we term the current method "near optimal", although true optimality can not be proved.

3.2. Asymptotic distribution. We now discuss the asymptotic distributions of the near optimal samples. For a set of $m$ points $\Theta_{m}^{S}$ selected by (3.2), its empirical distribution is

$$
\nu_{m}(x):=\frac{1}{m} \sum_{z \in \Theta_{m}^{S}} \delta_{z}(x)
$$

We are interested in the asymptotic limit of $\nu_{m}$ as $m \rightarrow \infty$.

In order to discuss the asymptotic limit, we first recall the definition of the $\mathcal{S}$ criterion (2.20) for matrice with infinite number of rows.

Definition 3.1 ([20]). Let $A_{\infty, p}$ be a matrix of size $\infty \times p$ and $S_{k}$ be a k-row selection matrix, where $k>p$. Then

$$
\mathcal{S}\left(A_{\infty, p}\right):=\liminf _{k \rightarrow \infty}\left(\sup _{S_{k}} \mathcal{S}\left(S_{k} A_{\infty, p}\right)\right) .
$$

Let us restrict to the total degree polynomial space (2.4) and use the subscript $n$ to explicitly denote the degree. We also recall the following theorem from [20].

Theorem 3.2 (Theorem 4.3 in [20]). Assume that for each degree $n \geq 1$, there exists a candidate set of infinite points $\Theta_{\infty, n}$, from which a model matrix $A_{\infty, p_{n}}$ can be constructed such that $\left.\mathcal{S}\left(A_{\infty, p_{n}}\right)\right)=1$. Here $p_{n}=\left(\begin{array}{c}n+d \\ d\end{array}\right)$ is the cardinality of the polynomial space. Then, there exists a sequence of point sets $\left\{\Theta_{k, n}\right\}_{k \geq p_{n}}$ with model matrix $A_{k, p_{n}}$ such that

$$
\left.\lim _{k \rightarrow \infty} \mathcal{S}\left(A_{k, p_{n}}\right)\right)=1
$$

Let $\nu_{k, n}$ be the empirical measure of $\Theta_{k, n}$. Then, for the sequence of measures $\left\{\nu_{k, n}\right\}_{k \geq p_{n}}, n \in \mathbb{N}$, there exists a convergent subsequence $\left\{\nu_{k_{i}, n}\right\}_{i \geq 1}$ such that

$$
\nu^{*}(x)=\lim _{n \rightarrow \infty} \lim _{i \rightarrow \infty} \nu_{k_{i}, n}(x)=\frac{d \mu}{d x}
$$

where $\mu$ is the measure in the orthogonality relation (2.6).

3.2.1. Bounded domain. For bounded domains, the following result holds.

THEOREM 3.3. Suppose that for each $n$, there exists a candidate set of infinite points $\Theta_{\infty, n}$ with model matrix $A_{\infty, p_{n}}$ such that $\left.\mathcal{S}\left(\sqrt{W} A_{\infty, p_{n}}\right)\right)=1$. Then there exists a sequence $\left\{\Theta_{k, n}\right\}_{k>p_{n}}$ with empirical measure $\nu_{k, n}$, which contains a subsequence $\nu_{k_{l}, n}$ that converges to measure $\nu_{n}^{*}$ satisfying

$$
\lim _{l \rightarrow \infty} \int_{D} \psi_{i}(x) \psi_{j}(x) \nu_{k_{l}, n}(x) d x=\int_{D} \psi_{i}(x) \psi_{j}(x) \nu_{n}^{*}(x) d x=\delta_{i j} .
$$


The sequence $\left\{\nu_{n}^{*}\right\}$ converges to $v$, i.e.,

$$
\lim _{n \rightarrow \infty} \nu_{n}^{*}(x)=v(x)=\frac{d \mu_{D}}{d x}, \quad \text { a.e. }
$$

where $d \mu_{D}$ is the equilibrium measure of $D$.

Proof. From Theorem 3.2 and (3.7), we have

$$
\delta_{i j}=\int_{D} \psi_{i}(x) \psi_{j}(x) \nu_{n}^{*}(x) d x=\int_{D} \phi_{i}(x) \phi_{j}(x) \omega_{n}(x) d x,
$$

where

$$
\omega_{n}(x)=\lambda_{n}(x) \nu_{n}^{*}(x)
$$

From (2.9) and the uniqueness of orthogonal polynomial probability measure,

$$
\omega(x)=\lim _{n \rightarrow \infty} \omega_{n}(x)=\lim _{n \rightarrow \infty} \lambda_{n}(x) \nu_{n}^{*}=\frac{\omega(x)}{v(x)} \nu^{*}(x), \quad \text { a.e. }
$$

Therefore, $\nu^{*}=\lim _{n \rightarrow \infty} \nu_{n}^{*}=v$.

Numerical tests were conducted to verify the theoretical estimates. Here we present the results obtained by Legendre polynomial, Chebyshev polynomial, and Jacobi polynomials with parameters $(\alpha, \beta)=(1,1)$ and $(0,2)$. The near optimal points are computed using the greedy algorithm from [20] over a candidate set of 100,000 uniformly distribution points in $[-1,1]$. On the left of Figure 3.1, we observe that at polynomial order $n=100$, the empirical distributions of the points from the four different orthogonal polynomials are all close to the asymptotic distribution. The maximum norm difference between the empirical distributions and the asymptotic distribution are shown in Fig. 3.1, for increasing order of polynomials. Algebraic convergence can be observed for all four different cases.
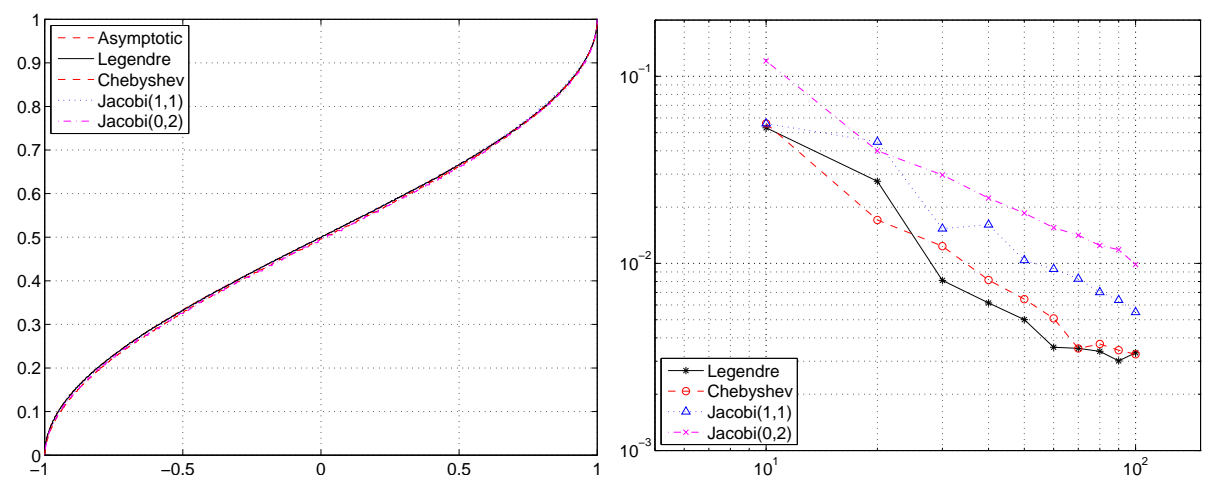

FIG. 3.1. Empirical distribution of the near optimal set for over-determined system. Left: the empirical measures at order $n=100$ for four different type of orthogonal polynomials; Right: Maximum norm differences between the empirical measures and the asymptotic distribution at increasing degrees of orthogonal polynomials.

3.2.2. Unbounded domain. Let us first recall a few results of the Christoffel function from the theory of orthogonal polynomials and the weighted potential theory. 
Let $D$ be an unbounded domain with a pluripotential-theoretic weight function $w$. For each polynomial degree $n \in \mathbb{N}$, let $\phi_{i}^{(n)}(x)$ be the family orthonormal under $w_{n}$, i.e.,

$$
\int_{D} \phi_{i}^{(n)}(x) \phi_{j}^{(n)}(x) w^{(n)}(x) d x=\delta_{i j} .
$$

For the whole space $\mathbb{R}^{d}$ with $w(x)=\exp \left(-\|x\|_{2}^{2}\right)$, the corresponding Hermite polynomial satisfies $h_{i}^{(n)}(x)=n^{\frac{d}{4}} h_{i}(\sqrt{n} x)$. For the tensor half space $[0, \infty)^{d}$ with $w(x)=$ $\exp \left(-\|x\|_{1}\right)$, the corresponding Laguerre polynomial satisifies $L_{i}^{(n)}(x)=n^{\frac{d}{2}} L_{i}(n x)$. Thus, we can write these as $\phi_{i}^{(n)}(x)=n^{d / 2 t} \phi_{i}\left(n^{1 / t} x\right)$, where the homogeneity exponent $t=2$ for the whole space and $t=1$ for the tensor half space. Then the reproducing kernel associated with the total degree polynomial space of degree $n$ with $w^{n}$ is defined by

$$
K_{n}^{(n)}=\sum_{0 \leq i \leq n}\left(\phi_{i}^{(n)}(x)\right)^{2}
$$

Then the following result is known.

Theorem 3.4. [[3, 4, 5]] Let $D \subset \mathbb{R}^{d}$ be an unbounded convex cone with $w$. Then

$$
\lim _{n \rightarrow \infty} \frac{1}{p} w^{n}(x) K_{n}^{(n)}(x)=v(x)=\frac{d \mu_{D}}{d x} .
$$

We are now in position to present the asymptotic distribution in unbounded domains.

THEOREM 3.5 (Unbounded). Suppose that for each n, there exists a candidate set of infinite points $\bar{\Theta}_{\infty, n} \subset S_{w}$ such that the model matrix $A_{\infty, p_{n}}$ using the scaled point set $n^{1 / t} \bar{\Theta}_{\infty, n}$ satisfies $\left.\mathcal{S}\left(\sqrt{W} A_{\infty, p_{n}}\right)\right)=1$. Here $p_{n}=\left(\begin{array}{c}n+d \\ d\end{array}\right)$ is the cardinality of the polynomial space. Then there exists a sequence $\left\{n^{1 / t} \bar{\Theta}_{k, n}\right\}_{k>p_{n}}$ with empirical measure $\left\{\nu_{k, n}\right\}_{k>p_{n}}$, which contains a convergent subsequence $\left\{\nu_{k_{l}, n}\right\}_{l \geq 1}$ that converges to measure $\nu_{n}^{*}$ satisfying

$$
\lim _{l \rightarrow \infty} \int_{D} \psi_{i}(x) \psi_{j}(x) \nu_{k_{l}, n}(x) d x=\int_{D} \psi_{i}(x) \psi_{j, n}(x) \nu_{n}^{*}(x) d x=\delta_{i j} .
$$

Let $\mu_{n}^{*}(z):=n^{d / t} \nu_{n}^{*}\left(n^{1 / t} z\right)$. Then, the sequence $\left\{\mu_{n}^{*}\right\}_{n \geq 1}$ converges to the weighted equilibrium measure $d \mu_{D}$, i.e.,

$$
\lim _{n \rightarrow \infty} \mu_{n}^{*}(z)=v(z)=\frac{d \mu_{D}}{d z}, \quad \text { a.e. }
$$

Proof. From the assumption that for each $n$, there exists a candidate set of infinite points $\bar{\Theta}_{\infty, n} \subset S_{w}$ such that $\left.\mathcal{S}\left(\sqrt{W} A_{\infty, n}\right)\right)=1$. By Theorem 3.2, we have

$$
\delta_{i j}=\int_{D} \psi_{i, n}(x) \psi_{j, n}(x) \nu_{n}^{*}(x) d x=\int_{D} \phi_{i}(x) \phi_{j}(x) \frac{p_{n}}{K_{n}(x)} \nu_{n}^{*}(x) d x .
$$

By setting $w_{n}(x):=\frac{p_{n}}{K_{n}(x)} \nu_{n}^{*}(x)$, we can write

$$
\delta_{i j}=\int_{D} \phi_{i}(x) \phi_{j}(x) w_{n}(x) d x .
$$


It then follows from the uniqueness of the orthogonal probability measure that

$$
\lim _{n \rightarrow \infty} \frac{p_{n}}{K_{n}(x)} \nu_{n}^{*}(x)=\lim _{n \rightarrow \infty} w_{n}(x)=w(x) .
$$

Substituting the change of variable $x=n^{1 / t} z$ into the measure $\nu_{n}^{*}(x) d x$ produces

$$
\nu_{n}^{*}(x) d x=n^{d / t} \nu_{n}^{*}\left(n^{1 / t} z\right) d z=\mu_{n}^{*}(z) d z, \quad \text { where } \quad \mu_{n}^{*}(z):=n^{d / t} \nu_{n}^{*}\left(n^{1 / t} z\right) .
$$

From Theorem 3.4, we have

$$
\lim _{n \rightarrow \infty} \frac{K_{n}^{(n)}(z) w^{n}(z)}{p_{n}}=\frac{d \mu_{D}}{d z} .
$$

Therefore by combining (3.17) and (3.18), it follows

$$
\lim _{n \rightarrow \infty} \frac{p_{n}}{K_{n}(x) w(x)} \nu_{n}^{*}(x)=\lim _{n \rightarrow \infty} \frac{p_{n}}{K_{n}^{(n)}(z) w^{n}(z)} \mu_{n}^{*}(z)=1 .
$$

This implies

$$
\lim _{n \rightarrow \infty} \mu_{n}^{*}(z)=\frac{d \mu_{D}}{d z}
$$

3.3. Greedy algorithm. The computation of the near optimal samples requires the selection of $m$ rows out of the $M$ rows of the model matrix $\sqrt{W} A_{M}$ such that (3.2) is optimized. This is a NP-hard problem and can not be easily solved. To circumvent the difficulty, we employ a greedy algorithm to select one row at a time such that (3.2) is optimized at each selection step. This is the similar algorithm for computing the S-optimal subset in [20].

Let $\mathcal{I}_{k}=\left\{i_{1}, \ldots, i_{k}\right\}$ be a sequence consisting of $k$ unique indices, where $1 \leq$ $i_{j} \leq M$ for all $j=1, \ldots, k$, and $i_{j} \neq i_{l}$ for any $j \neq l$. Also, let $\sqrt{W} A_{\mathcal{I}_{k}}=$ $\left[\sqrt{w_{i_{1}}} A_{\left(i_{1}\right)} ; \ldots ; \sqrt{w_{i_{k}}} A_{\left(i_{k}\right)}\right]$ denote the matrix constructed by the $\mathcal{I}_{k}$ rows out of the $\sqrt{W} A_{M}$. Then, the near optimal points can be obtained by selecting the row indices such that

$$
\mathcal{I}_{m}^{\dagger}=\underset{\mathcal{I}_{m}}{\operatorname{argmax}} \mathcal{S}\left(\sqrt{W} A_{\mathcal{I}_{m}}\left(\Theta_{M}\right)\right)
$$

By using this index selection format, the greedy algorithm is as follows.

Given the current selection of $k \geq p$ rows, which results in a index sequence $\mathcal{I}_{k}=\left\{i_{1}, \ldots, i_{k}\right\}$, find the next row index such that

$$
i_{k+1}=\underset{i \in \mathcal{I}_{M} \backslash \mathcal{I}_{k}}{\operatorname{argmax}} \mathcal{S}\left(\sqrt{W} A_{\mathcal{I}_{k} \cup\{i\}}\right), \quad \mathcal{I}_{k+1}=\mathcal{I}_{k} \cup\left\{i_{k+1}\right\},
$$

where $\mathcal{I}_{M}=\{1, \ldots, M\}$ is the set of all indices.

Since the quantity $\mathcal{S}$ is defined only for matrices of size $k \times p$ with $k \geq p$, the greedy algorithm requires specification of the initial $p$ points to start. Here we discuss two choices to determine the initial $p$ points. 
3.3.1. Initial $p$ points using truncated square matrices. The first choice is to apply the same greedy algorithm (3.20) to truncated matrices of size $k \times k$ when $k<p$. This obviously requires the truncation of columns of the original $\sqrt{W} A_{M}$. It is the same procedure adopted in [20]. This algorithm starts with an initial choice of index $i_{1}$, which can be a random choice. It stops when $k=p$ and resumes the standard greedy algorithm (3.20).

3.3.2. Initial $p$ points using approximate Fekete points. Another choice is to use the concept of Fekete points, which is used in polynomial interpolation theory. The Fekete points seek to maximize the determinant of the Vandermonde-like matrix. Using the same principle, we can find the first $p$ indices $\mathcal{I}_{p}$ which maximize the determinant of the $p \times p$ square matrix $\sqrt{W} A_{\mathcal{I}_{p}}^{s q}$, i.e.,

$$
\mathcal{I}_{p}=\underset{\mathcal{I}_{p} \subset \mathcal{I}_{M}}{\operatorname{argmax}}\left|\operatorname{det} \sqrt{W} A_{\mathcal{I}_{p}}^{s q}\right| .
$$

The motivation for using the Fekete points is that it is known that the asymptotic distribution of the Fekete points in bounded domain is the asymptotic measure [3]. This is the same property as the near optimal samples from Theorem 3.3 and 3.5. More importantly, the Fekete points can be easily computed using a greedy algorithm, resulting in the approximate Fekete points (AFP) [6]. The algorithm for computing the AFP is remarkably simple. This results in a much reduced computational effort for determine the initial $p$ points. Our numerical examples demonstrate that the use of the AFP as the initial $p$ points results in negligible difference in the final result, with the use of the square matrices approach slightly better.

4. Numerical Examples. In this section we present numerical examples to demonstrate the performance of the near optimal sampling strategy. We first examine the stability of the model matrix and then provide approximation results for a selection of functions in multiple dimensions. In all the figures, we use "CLS" to stand for the Christoffel least squares method by [17], "S-optimality" for the quasi-optimal subset selection method by [20]. For the nearly optimal sampling method presented in this paper, we use "nOPT", where the initial $p$ points are determined by the truncated square matrices approach (Section 3.3.1), and "nOPT-F" where the initial $p$ points are determined by the approximate Fekete points (Section 3.3.2).

In all examples we use the total degree polynomial spaces (2.4) to construct the polynomial basis functions. We use $\alpha$ to denote the sampling rate, i.e., $m=\alpha \cdot p$. We focus on the linear and low oversampling ratios $\alpha=1.2$, and also $\alpha=2$, to demonstrate the effectiveness of the method.

4.1. Matrix stability. We first numerically investigate the condition number of the model matrix resulted from the sampling selection

$$
\kappa(\sqrt{W} A)=\frac{\sigma_{\max }(\sqrt{W} A)}{\sigma_{\min }(\sqrt{W} A)},
$$

where $\sigma_{\max }$ and $\sigma_{\min }$ are the maximum and minimum singular values of the matrix, respectively. To reduce the effect of statistical fluctuation, we report the statistical average of the condition numbers over 50 ensemble of independent tests.

4.1.1. Bounded domain. Without loss of generality, we consider $[-1,1]^{d}$ hypercube and use Legendre polynomials. In Fig. 4.1, the condition numbers in $d=2$ with respect to increasing polynomial degree from $n=5$ to $n=35$ are shown. It 
can be seen that at the very low over-sampling rate of $\alpha=1.2$, both the CLS and the S-optimal points exhibit weak instability. The current near optimal sampling method, however, exhibits much better stability, almost as good as the CLS reference solutions, obtained by oversampling $1.5 p \log p$, which is proven to be stable. Similar results for $d=4$ can be seen from Fig. 4.2 .

The condition numbers of the standard Monte Carlo method using the corresponding uniform random samples are not shown. It is known to be unstable and the condition numbers become so large that they skew the scale of the figures out of proportion.
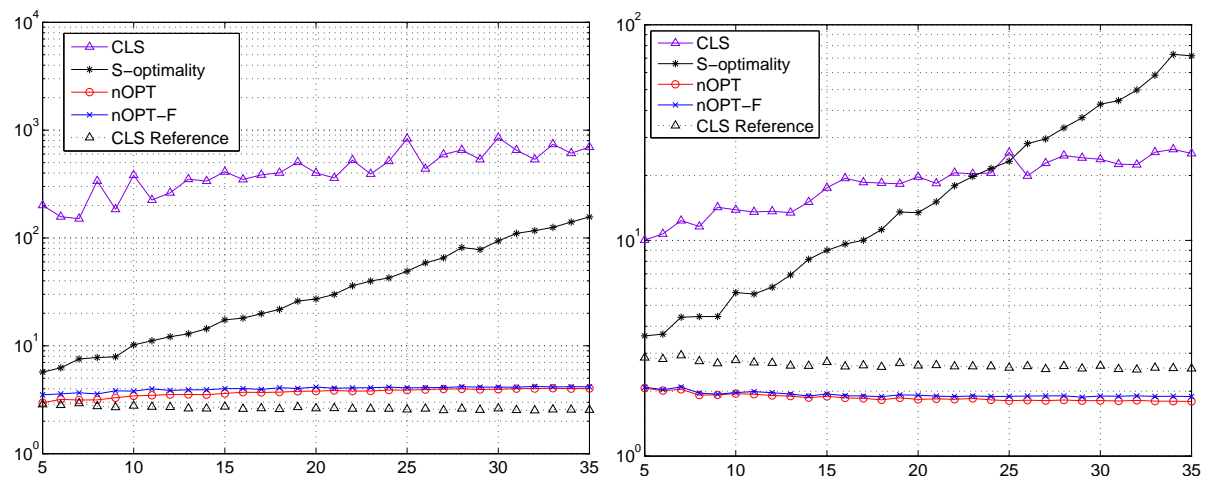

FIG. 4.1. $d=2$, Legendre polynomial. The condition numbers of the model matrix at increasing polynomial degree from 5 to 35. Left: oversampling rate $\alpha=1.2$; Right: oversampling rate $\alpha=2$.
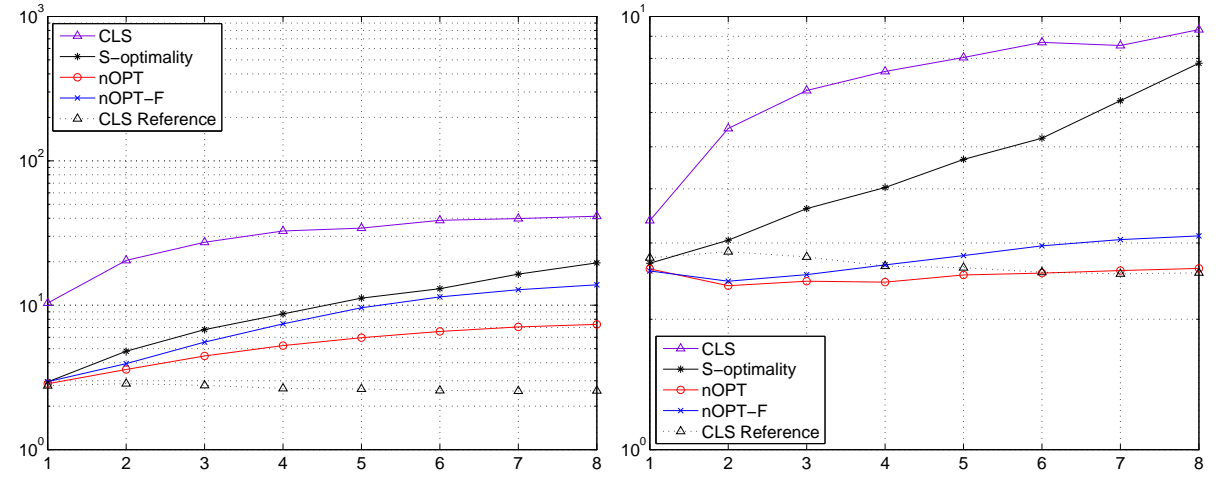

FIG. 4.2. $d=4$, Legendre polynomial. The condition numbers of the model matrix at increasing polynomial degree from 1 to 8 . Left: oversampling rate $\alpha=1.2$; Right: oversampling rate $\alpha=2$.

4.1.2. Unbounded domain. For unbounded domain case, we first consider the whole space $\mathbb{R}^{d}$ with the Gaussian density function $\exp \left(-\sum\|z\|_{2}^{2}\right)$. The Hermite orthogonal polynomials are use as the basis functions. In Figure 4.3, we report the condition numbers with respect to the increasing polynomial degree in both the $d=2$ and $d=4$. The results of $\alpha=1.2$ are shown as solid lines and those of $\alpha=2$ are shown as dashed lines. Again, the reference solutions are obtained by CLS with $\alpha=\log p$ which is proven to be stable. Similar to the case of bounded domain, we observe much improved the stability property by the current method "nOPT" and "nOPT-F".

We then consider the "half-space" of $[0, \infty)^{d}$ with an exponential density function 
$\exp \left(-\|z\|_{1}\right)$. The Laguerre orthogonal polynomials are used as the basis functions. The condition numbers are plotted in Figure 4.4. Again, we observe much improved stability property of the current method at such low and linear oversampling rate. It is as good as the mathematically stable solution obtained by CLS using $p \log p$ samples.
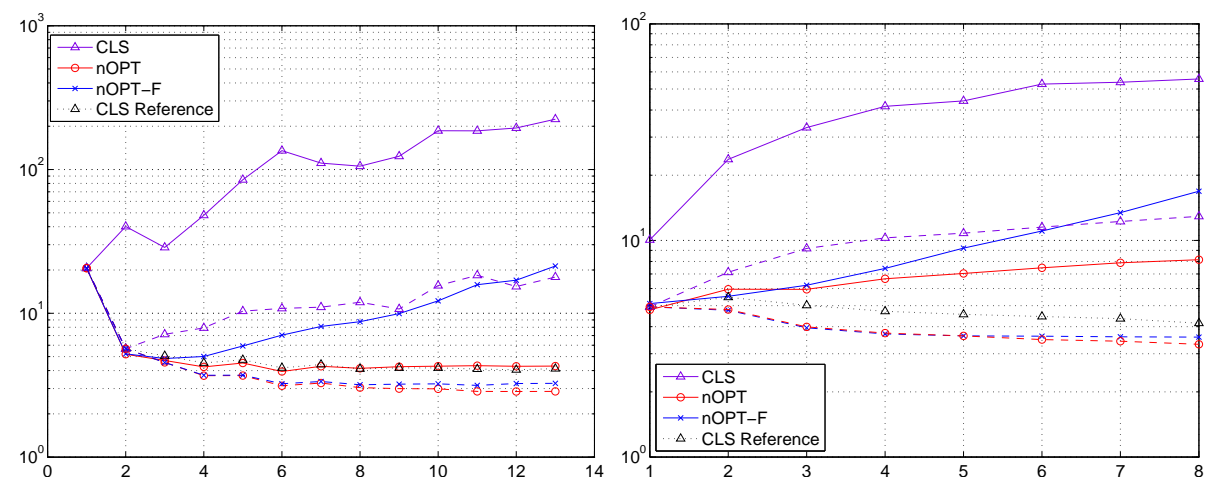

FIG. 4.3. Conditions numbers of Hermite polynomial of increasing degree with oversampling rate $\alpha=1.2$ (solid lines) and $\alpha=2$ (dashed lines). Left: $d=2 ;$ Right: $d=4$.
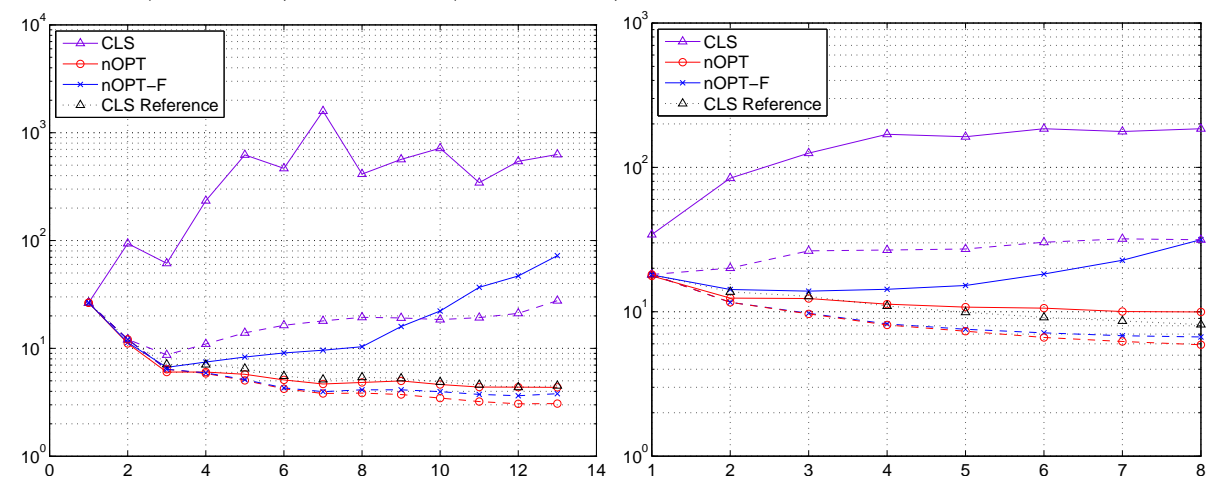

FIG. 4.4. Condition numbers of Laguerre polynomial of increasing degree with oversampling rate $\alpha=1.2$ (solid lines) and $\alpha=22$ (dashed lines). (Left: $d=2$; Right: $d=4$.

4.2. Function approximation. We now examine the performance of our proposed strategy in term of function approximation. We adopt a variety of target functions from Genz [10], which have been widely used for multi-dimensional function 
integration and approximation tests. More specifically, the test functions include

$$
\begin{aligned}
& f_{1}=\exp \left(-\sum_{i=1}^{d} c_{i}^{2}\left(\frac{x_{i}+1}{2}-w_{i}\right)^{2}\right) ; \\
& f_{2}=\exp \left(-\sum_{i=1}^{d} c_{i}\left|\frac{x_{i}+1}{2}-w_{i}\right|\right) ; \\
& f_{3}=\left(1+\sum_{i=1}^{d} c_{i} \frac{\left(x_{i}+1\right)}{2}\right)^{-(d+1)}, \text { where } c_{i}=\frac{1}{i^{2}} \quad \text { (Corner peak); } \\
& f_{4}=\prod_{i=1}^{d}\left(c_{i}^{-2}+\left(\frac{x_{i}+1}{2}-w_{i}\right)^{2}\right)^{-1} \quad \text { (Product peak). }
\end{aligned}
$$

Here, $c=\left(c_{1}, \cdots, c_{d}\right)$ are parameters controlling the difficulty of the functions, and $w=\left(w_{1}, \cdots, w_{d}\right)$ are shifting parameters. We also employ, exclusively for $d=2$, the Franke function $([9])$

$$
\begin{aligned}
f & =0.75 \exp \left(-\frac{\left(9 x_{1}-2\right)^{2}}{4}-\frac{\left(9 x_{2}-2\right)^{2}}{4}\right)+0.75 \exp \left(-\frac{\left(9 x_{1}+1\right)^{2}}{49}-\frac{9 x_{2}+1}{10}\right) \\
& +0.5 \exp \left(-\frac{\left(9 x_{1}-7\right)^{2}}{4}-\frac{\left(9 x_{2}-3\right)^{2}}{4}\right)-0.2 \exp \left(-\left(9 x_{1}-4\right)^{2}-\left(9 x_{2}-7\right)^{2}\right) .
\end{aligned}
$$

To evaluate the performance of the approximation, we compute the numerical errors at 40,000 Monte Carlo random points. These points are generated independently after the simulations. We report the $\ell_{2}$ norm of the numerical errors over these points.

4.2.1. Bounded domain results. We first consider the two dimensional case $d=2$ on $[-1,1]^{2}$ and seek approximations using Legendre polynomials. The polynomial degree at is fixed $n=10$. At $d=2, p=\operatorname{dim} \mathcal{P}_{n}^{d}=66$. Thus, oversampling starts at $m=67$. The numerical errors of the different methods for the four test functions in (4.1) are shown in Fig. 4.5, with respect to the number of sample points. We observe that all methods show decay of errors when the number of samples is increased, as expected. The current near optimal sampling methods, using either the squared matrices approach (nOPT) or the Fekete point approach (nOPT-F) for the initial $p$ points, deliver very similar results. The results by the S-optimal points from [20] are also very similar. All of these are notably better than the CLS from [17]. Since the current near optimal method is a combination of both [17] and [20], these results suggest that the high accuracy is mostly due to the strategy by [20]. The references solution here are obtained by CLS using $p \log p$ points.

Error behavior against the polynomial degree is also investigated. Here we present the results for the Franke function (4.2). On the left of Fig. 4.6, we plot the errors obtained at degree $n=20$, which results in $p=\operatorname{dim} \mathcal{P}_{n}^{d}=231$. Very similar results by the different methods are observed. On the right of Fig. 4.6, the errors with respect to increasing polynomial degrees from $n=5$ to $n=20$ are plotted. Here, all methods use a very low oversampling rate of $\alpha=1.1$, except that the reference solution is obtained by $p_{n} \log p_{n}$ points. We again notice the small errors by the S-optimal points ([20]) and the two versions of the current near optimal points, the latter of which are slightly better. 

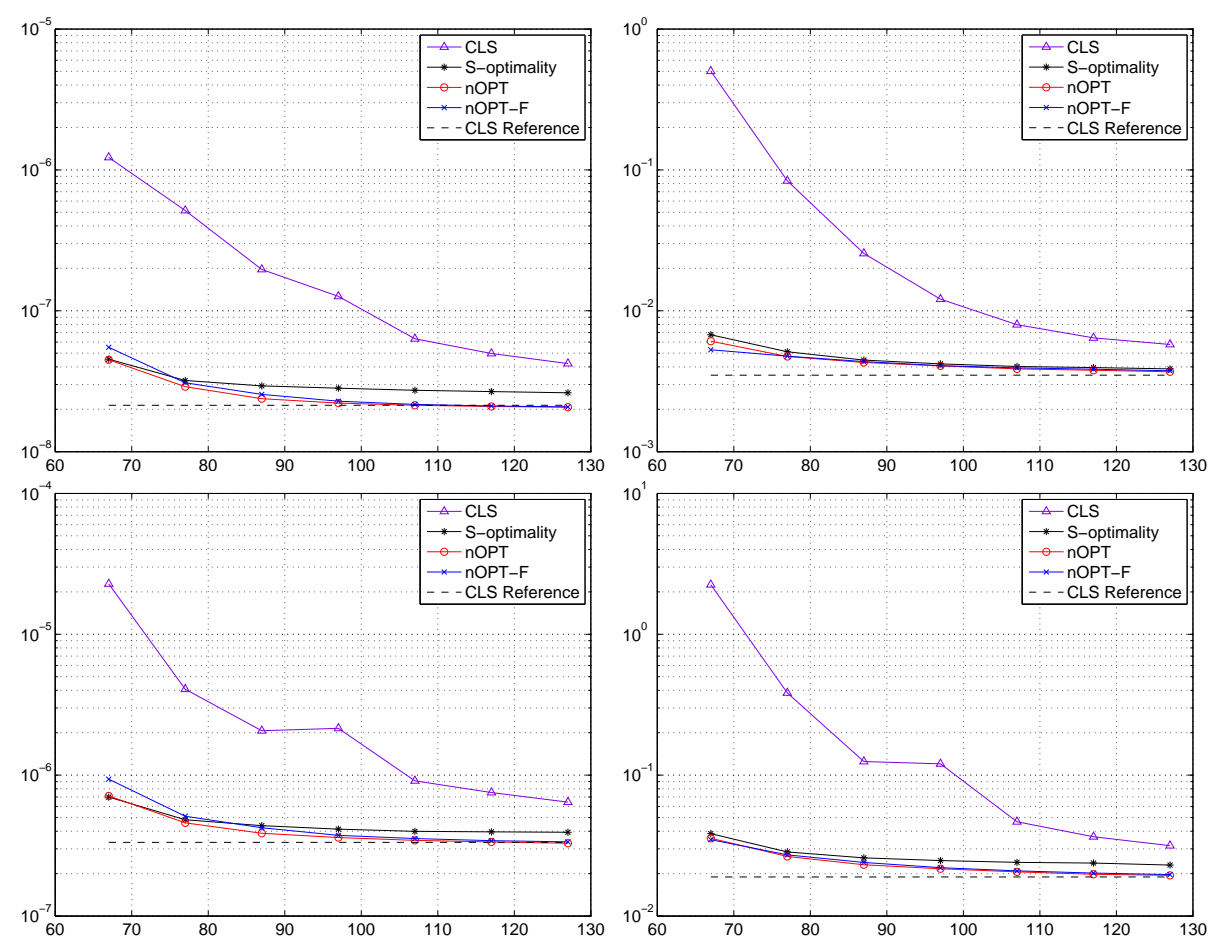

FIG. 4.5. Errors versus number of sample points by Legendre polynomials for the four test functions (4.1) at $d=2$ and $n=10$. Top left: $f_{1}$ with $c=(1,1)$ and $w=(1,0.5)$; Top right: $f_{2}$ with $c=(-2,1)$ and $w=(0.25,-0.75)$; Bottom left: $f_{3}$; Bottom right: $f_{4}$ with $c=(-3,2)$ and $w=(0.5,0.5)$. Dashed lines are the best errors obtained by the reference solutions.
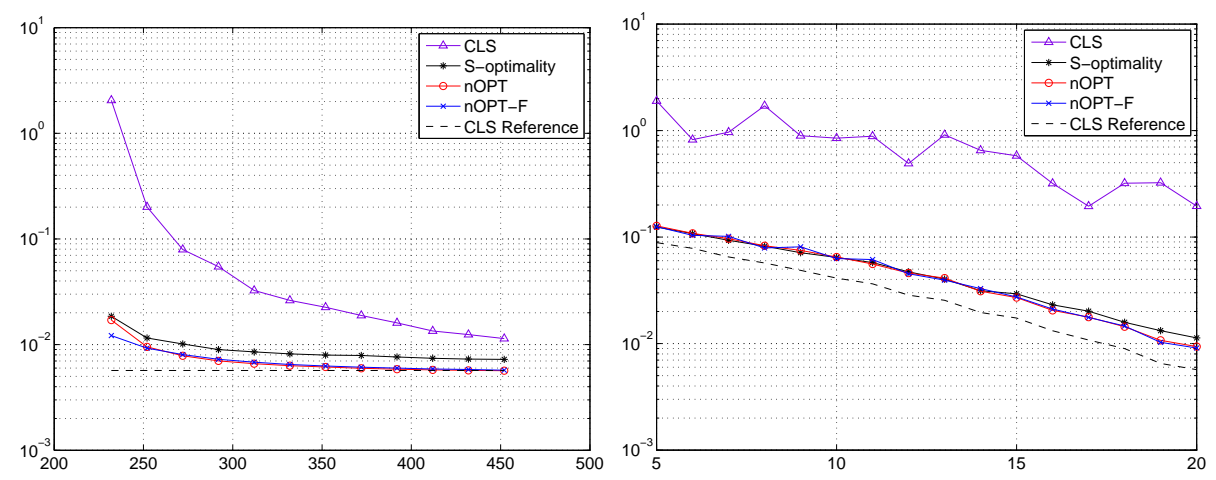

FIG. 4.6. Left: Errors versus number of sample points by Legendre polynomials at $d=2$ and $n=20$ for the Franke function (4.2). Right: Errors versus polynomial degrees at the oversampling rate of 1.1. The dashed line is the best error obtained by the reference solution.

We now present a higher dimensional case of $d=4$. The results for the target function $f_{3}$ and $f_{4}$ in $(4.1)$, in domain $[-1,1]^{d}$, are shown in Fig. 4.7. On the left of the figure, the error decay with respect to the number of samples at a fixed polynomial degree $n=8$ is plotted. On the right of the figure, the error decay with respect to increasing polynomial degree is plotted, at the oversampling rate $\alpha=1.1$. Similar conclusion as in $d=2$ can be drawn here. 

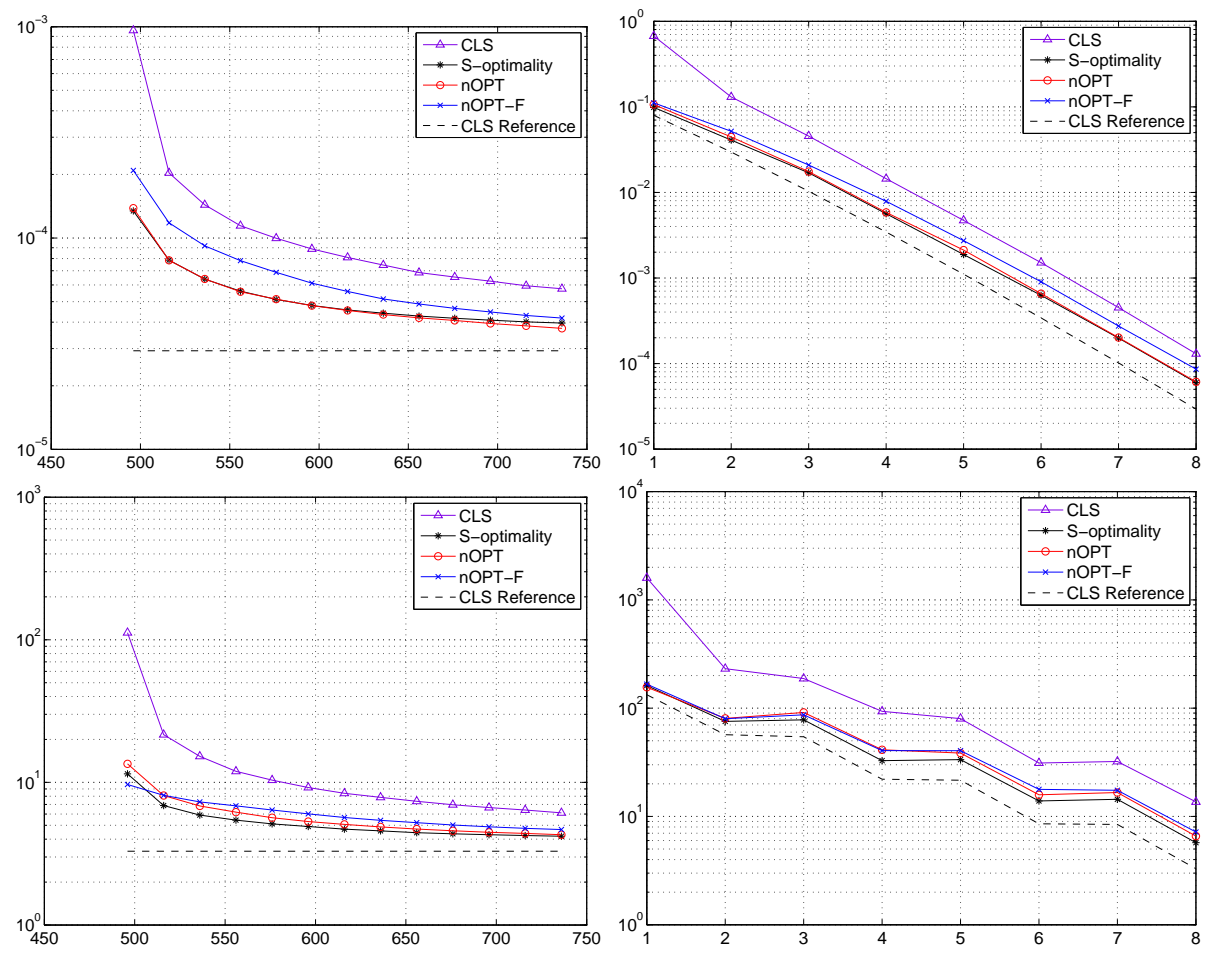

Fig. 4.7. Errors by Legendre polynomials in $d=4$. Top row: $f_{3}$; Bottom row: $f_{4}$ with $c=(1,2,3,4)$ and $w=(0.5,0.5,0.5,0.5)$. Left column: The errors versus number of sample points for $f_{3}$ and $f_{4}$ at $n=8$. Right column: The errors versus increasing polynomial degree from 1 to 8 with the oversampling rate $\alpha=1.1$. Dashed lines are the best errors obtained by the reference solutions.

4.2.2. Unbounded domain results. For the unbounded domain case, we consider the whole space in $d=3$ and use Hermite polynomials. We examine both the Corner peak function from (4.1) and the following exponential function

$$
f_{5}(x)=\exp \left(-\sum_{i=1}^{d} x_{i}\right) .
$$

In Figure 4.8, we show the errors with respect to increasing polynomial degree from $n=1$ to $n=10$ at the oversampling rate of 1.1. The reference solutions are obtained by CLS using oversampling of $\alpha=2$. The left plot shows the results of approximating $f_{5}(x)$ while the right plot shows the results for the Corner Peak. Again we observe that the current near optimal methods deliver very accurate performance.

4.3. High dimensional examples: stochastic collocation for SPDE. For high dimensional example, we consider the following stochastic diffusion problem in $d=10$ dimensional random space.

$$
-\frac{\partial}{\partial x}\left[\kappa(x, y) \frac{\partial u}{\partial x}(x, y)\right]=f, \quad(x, y) \in(0,1) \times \mathbb{R}^{d},
$$



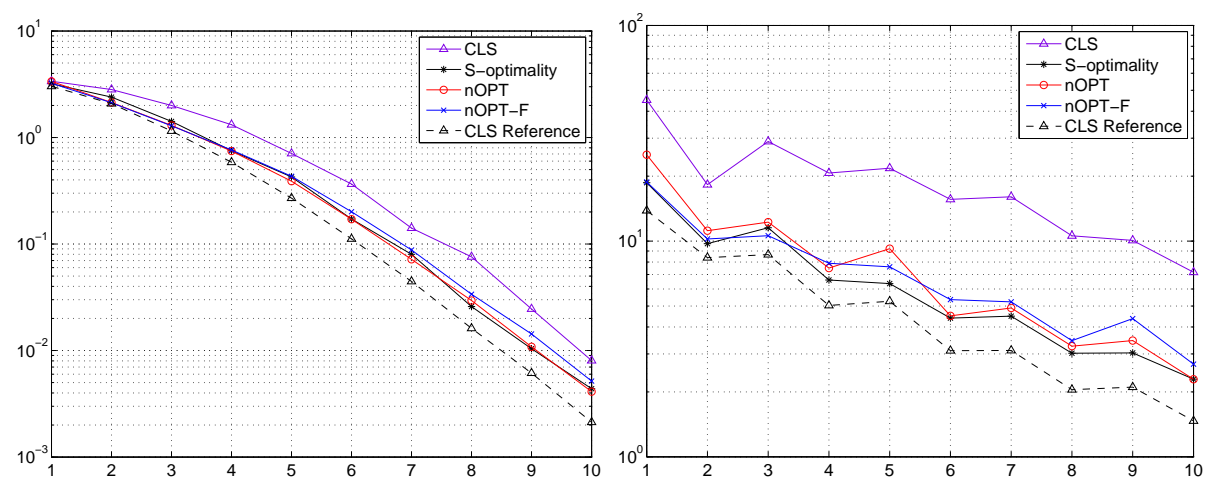

FIG. 4.8. Errors against polynomial degree, for $d=3$ whole space using Hermite polynomial. Left: $f(x)=\exp \left(-\sum_{i=1}^{d} x_{i}\right)$; Right: Corner Peak function with $(c)_{i}=i$ and $(w)_{i}=0.5$. The dashed lines is the best errors obtained by the reference solutions.

with forcing term $f=$ const and boundary conditions

$$
u(0, y)=0, \quad u(1, y)=0, \quad y \in \mathbb{R}^{d} .
$$

We assume that the random diffusivity take an analytical form (for benchmarking purpose)

$$
\kappa(x, y)=1+\sigma \sum_{k=1}^{d} \frac{1}{k^{2} \pi^{2}} \cos (2 \pi k x) y_{k},
$$

where $y=\left(y_{1}, \cdots, y_{d}\right)$ is a random vector with independent and identically distributed components.

For the numerical test, we set $f=2, \sigma=1, d=10$ and let $y$ be uniformly distributed in $[-1,1]^{10}$. For comparison, we also employ generalized polynomial chaos (gPC) ([23]) Galerkin methdo using Legendre polynomials. Note that the gPC Galerkin method solves exactly $p=\operatorname{dim} \mathbb{P}_{n}^{d}$ number of equations and exhibits exponential error convergence in this case. However, the resulting deterministic equations for the expansion coefficients are coupled and require care to solve. The properties of the Galerkin system and generalized polynomial chaos (gPC) are well studied and documented in a large amount of literature. Interested readers can consult [22] for a quick review. At expansion order $n=4$, the gPC Galerkin solution induces negligible errors and is be used as the reference solution.

In Fig. 4.9, the error convergence with respect to the polynomial degree is shown. It can be seen that the near optimal sampling strategy results in exponential error convergence, like the gPC Galerkin method. The error by the S-optimal points from [20] also exhibits a similar behavior. Note that in all the cases, we only oversample by 10 points, i .e., $m=p+10$. This makes the nonintrusive nOPT, as well as S-optimality, more attractive than the gPC Galerkin.

In Fig. 4.10, the error convergence of the current nOPT method is shown with respect to the number of samples, at different polynomial orders. At each polynomial order $n=1,2,3,4$, the number of samples starts from $\left(\operatorname{dim} \mathbb{P}_{n}^{d}+1\right)$. It can be seen that the results by nOPT has very similar accuracy, compared to gPC Galerkin, and the number of samples is only marginally larger than $p=\operatorname{dim} \mathbb{P}_{n}^{d}$. Since the gPC Galerkin solves the $p$ number of couple equations, the nOPT represents a convenient 


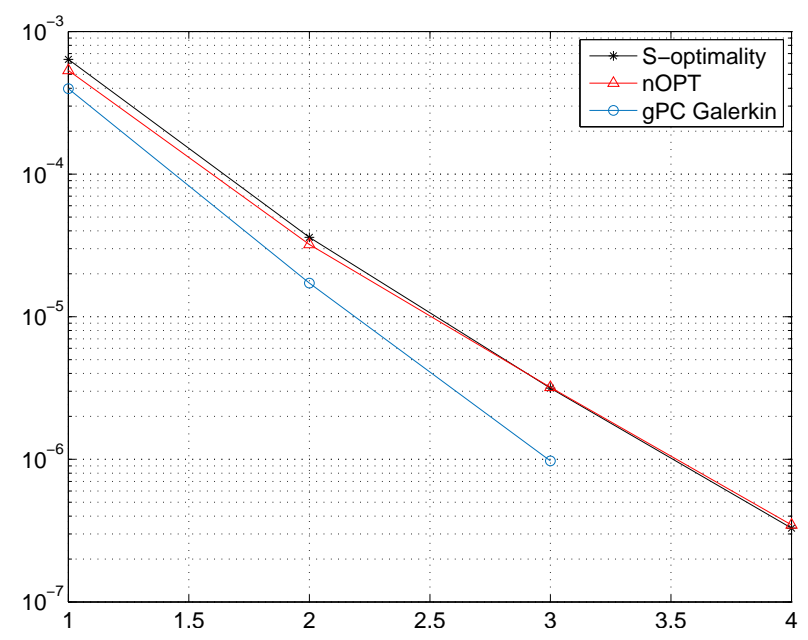

FIG. 4.9. Error convergence versus Legendre polynomial expansion degree at dimension $d=10$. Both S-optimality and nOPT are obtained by oversampling 10 points, i.e., $m=\operatorname{dim} \mathbb{P}_{n}^{d}+10$. The $g P C$ Galerkin solution at $n=4$ is used as the reference solution.

alternative. It also can be seen that in this case, the nOPT has slightly better accuracy than the S-optimal points, especially at lower orders.

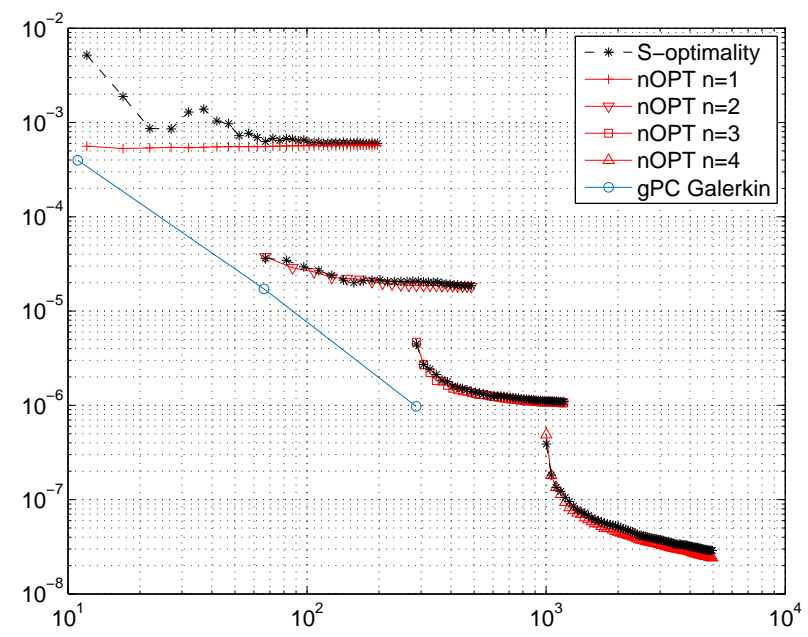

FIG. 4.10. Error convergence versus the number of equations to solve, at different Legendre expansion degree $n=1,2,3,4$ and dimension $d=10$. The $g P C$ Galerkin solution at $n=4$ is used as the reference solution.

5. Conclusion. In this paper, we presented near optimal strategy for leastsquares polynomial regression. The method combines the idea of Christoffel least squares method ([17]), which offers the almost optimal stability property, and the idea of S-optimality design ([20]), which offers the almost optimal accuracy at any given number of samples. Consequently, the new method offers superb stability and accuracy for arbitrarily smaller number of samples, as long as it is in the over-determined case. This can be a useful tool for pratical polynomial regression when the data 
sample collection is expensive or difficult.

Acknowledgment. This work is in part supported by AFOSR, DARPA, and NSF.

\section{REFERENCES}

[1] A.C. Atkinson, A.N. Donev, and R.D. Tobias. Optimum Experimental Designs, with SAS. Oxford Univ. Press, 2007.

[2] R.A. Bailey. Design of Comparative Experiments. Cambridge Univ. Press, 2008.

[3] Robert Berman, Sébastien Boucksom, and David Witt Nyström. Fekete points and convergence towards equilibrium measures on complex manifolds. Acta Mathematica, 207(1):1-27, 2011.

[4] Robert J Berman. Bergman kernels and equilibrium measures for line bundles over projective manifolds. American journal of mathematics, 131(5):1485-1524, 2009.

[5] Robert J Berman. Bergman kernels for weighted polynomials and weighted equilibrium measures of cn. Indiana University mathematics journal, 58(4):1921-1946, 2009.

[6] Len Bos, Stefano De Marchi, Alvise Sommariva, and Marco Vianello. Computing multivariate fekete and leja points by numerical linear algebra. SIAM Journal on Numerical Analysis, 48(5):1984-1999, 2010.

[7] G.E. Box, J.S. Hunter, and W.G. Hunter. Statistics for Experimenters: Design, Innovation, and Discovery. Wiley, 2nd edition, 2005.

[8] A. Cohen, M. A. Davenport, and D. Leviatan. On the stability and accuracy of least squares approximations. Found. Comput. Math., 13(5):819-834, 2013.

[9] R. Franke. A critical comparison of some methods for interpolation of scattered data. Technical Report NPS-53-79-003, Naval Postgraduate School, March 1979.

[10] A. Genz. Testing multidimensional integration routines. In B. Ford, J.C. Rault, and F. Thomasset, editors, Tools, Methods, and Languages for Scientific and Engineering Computation, pages 81-94. North-Holland, 1984.

[11] P. Goos and B. Jones. Optimal Design of Experiments: A Case Study Approach. Wiley, 2011.

[12] L. Gyørfi, M. Kohler, A. Krzyżak, and H. Walk. A Distribution-Free Theory of Nonparametric Regression. Springer Series in Statistics, Springer-Verlag, Berlin, 2002.

[13] R.L. Mason, R.F. Gunst, and J.L. Hess. Statistical design and analysis of experiments with applications to engineering and science. 1989.

[14] G. Migliorati and F. Nobile. Analysis of discrete least squares on multivariate polynomial spaces with evaluations at low-discrepancy point sets. J. Complexity, in print, 2015.

[15] G. Migliorati, F. Nobile, E. von Schwerin, and R. Tempone. Approximation of quantities of interest in stochastic PDEs by the random discrete $\$ 1^{\wedge} 2 \$$ projection on polynomial spaces. SIAM Journal on Scientific Computing, 35(3):A1440-A1460, May 2013.

[16] G. Migliorati, F. Nobile, E. von Schwerin, and R. Tempone. Analysis of the discrete $L^{2}$ projection on polynomial spaces with random evaluations. Found. Comput. Math., 14(3):419-456, 2014.

[17] Akil Narayan, John D Jakeman, and Tao Zhou. A christoffel function weighted least squares algorithm for collocation approximations. arXiv preprint arXiv:1412.4305, 2014.

[18] Paul Nevai. Géza freud, orthogonal polynomials and christoffel functions. a case study. Journal of Approximation Theory, 48(1):3-167, 1986.

[19] F. Pukelsheim. Optimal Design of Experiments. SIAM, 2006.

[20] Y. Shin and D. Xiu. Nonadaptive quasi-optimal points selection for least squares linear regression. SIAM J. Sci. Comput., 38(1):A385-A411, 2016.

[21] C.F. Wu and M.S. Hamada. Experiments: planning, analysis, and optimization. John Wiley \& Sons, 2009.

[22] D. Xiu. Numerical methods for stochastic computations. Princeton Univeristy Press, Princeton, New Jersey, 2010.

[23] D. Xiu and G.E. Karniadakis. The Wiener-Askey polynomial chaos for stochastic differential equations. SIAM J. Sci. Comput., 24(2):619-644, 2002. 